\title{
Electron-Phonon beyond Fröhlich: Dynamical Quadrupoles in Polar and Covalent Solids
}

\author{
Guillaume Brunin $\odot,{ }^{1}$ Henrique Pereira Coutada Miranda $\odot,{ }^{1}$ Matteo Giantomassi $\odot,{ }^{1}$ Miquel Royo $\odot,{ }^{2}$ \\ Massimiliano Stengel@ ${ }^{2,3}$ Matthieu J. Verstraete $\oplus^{4,5}$ Xavier Gonze $\odot,{ }^{1,6}$ \\ Gian-Marco Rignanese $\mathbb{C}^{1}{ }^{1}$ and Geoffroy Hautier ${ }^{1}{ }^{1 *}$ \\ ${ }^{1}$ UCLouvain, Institute of Condensed Matter and Nanosciences (IMCN), Chemin des Étoiles 8, B-1348 Louvain-la-Neuve, Belgium \\ ${ }^{2}$ Institut de Ciència de Materials de Barcelona (ICMAB-CSIC), Campus UAB, 08193 Bellaterra, Spain \\ ${ }^{3}$ ICREA-Institució Catalana de Recerca i Estudis Avanćats, 08010 Barcelona, Spain \\ ${ }^{4}$ NanoMat/Q-Mat/CESAM, Université de Liège (B5), B-4000 Liège, Belgium \\ ${ }^{5}$ Catalan Institute of Nanoscience and Nanotechnology (ICN2), Campus UAB, 08193 Bellaterra, Spain \\ ${ }^{6}$ Skolkovo Institute of Science and Technology, Skolkovo Innovation Center, Nobel St. 3, 143026, Moscow, Russia
}

(Received 24 January 2020; revised 14 May 2020; accepted 20 August 2020; published 21 September 2020)

\begin{abstract}
We include the treatment of quadrupolar fields beyond the Fröhlich interaction in the first-principles electron-phonon vertex in semiconductors. Such quadrupolar fields induce long-range interactions that have to be taken into account for accurate physical results. We apply our formalism to Si (nonpolar), GaAs, and $\mathrm{GaP}$ (polar) and demonstrate that electron mobilities show large errors if dynamical quadrupoles are not properly treated.
\end{abstract}

DOI: 10.1103/PhysRevLett.125.136601

The electron-phonon $(e-p h)$ interaction plays a key role in the description of various physical phenomena (e.g., electronic transport, phonon-assisted light absorption, phononmediated superconductivity) [1]. In state-of-the-art ab initio methods, the $e$-ph coupling is described within density functional theory (DFT) by expanding the Kohn-Sham (KS) effective potential [2] in the nuclear displacements, while vibrational properties are obtained using density-functional perturbation theory (DFPT) $[3,4]$. This DFPT-based computational scheme enables the calculation of screened $e$-ph matrix elements at a microscopic level with ab initio quality [1]. However, accurate $e$-ph related properties require a description of the coupling on very dense reciprocal-space wave vector grids across the full Brillouin zone (BZ) thus making direct ab initio $e$-ph computations in real materials costly and sometimes even impracticable.

Despite formal differences in the treatment of the electron wave function, all approaches proposed so far rely on the spatial localization of the $e$-ph coupling to interpolate it with respect to the phonon wave vector $\mathbf{q}$ [5-8]. The fundamental physical assumption is that a relatively small real-space supercell can capture the full strength of the coupling. For metals this is trivial as screening lengths are very short. However, in semiconductors and insulators, the incomplete screening of the potential generated by the atomic displacements leads to long-range (LR) interactions. For polar materials, these interactions show up in the long-wavelength limit $(\mathbf{q} \rightarrow \mathbf{0})$ for instance in the LO-TO splitting of the optical frequencies [9] and the Fröhlich divergence of the $e$-ph matrix elements [10]. The analytical treatment of the LR dipole-dipole interaction to obtain a reliable longwavelength dynamical matrix was developed in the early days of DFPT [3,4]. More recently, the Fröhlich contribution to the LR $e$-ph matrix elements was generalized to anisotropic materials [6,11], opening up a first avenue for computations of $e$-ph effects in polar materials [1].

In this Letter, we go beyond these seminal contributions, demonstrate the importance of the next-to-leading order terms derived by Vogl [12], and explain their physical origin. In this new approach, the LR contributions are expressed in terms of the high-frequency dielectric tensor, Born effective charges (dipole potential), dynamical quadrupoles $[13,14]$ (quadrupole potential), and the response to a homogeneous static electric field (local-field potential, also quadrupolar) [3,4]. Despite their importance in describing certain piezoelectric properties $[13,15]$, quadrupolar interactions have never been considered in firstprinciples $e$-ph calculations. In nonpolar materials, dynamical quadrupoles represent the leading contribution to the LR potential. Even in polar materials, where the focus has been on the divergent Fröhlich interaction for LO modes [11], the quadrupolar field dominates the LR potential for the TO and acoustic modes [12].

We first describe the physical origin and LR nature of the quadrupolar interaction. We then demonstrate that substantial errors in carrier mobility in well-known polar and nonpolar semiconductors are introduced if the quadrupolar interaction is not properly accounted for. We show results for the classical semiconductors Si, GaAs, and GaP. As quadrupolar interactions have not been considered so far, our analysis indicates that many previous ab initio $e$-ph calculations for semiconductors should be critically reconsidered.

The key ingredients in $e$-ph computations are the coupling matrix elements $g_{m n \nu}(\mathbf{k}, \mathbf{q})=\left\langle\psi_{m \mathbf{k}+\mathbf{q}}\left|\Delta_{\mathbf{q} \nu} V\right| \psi_{n \mathbf{k}}\right\rangle$ with $\psi_{n \mathbf{k}}$ 
the $n \mathbf{k}$ Bloch state and $\Delta_{\mathbf{q} \nu} V$ the first-order variation of the KS potential $V$ due to a phonon mode of wave vector $\mathbf{q}$ and branch index $\nu$ [1]. The scattering potential is defined as

$$
\Delta_{\mathbf{q} \nu} V=\frac{1}{\sqrt{2 \omega_{\mathbf{q} \nu}}} \sum_{\kappa \alpha} \frac{e_{\kappa \alpha, \nu}(\mathbf{q})}{\sqrt{M_{\kappa}}} V_{\kappa \alpha, \mathbf{q}}(\mathbf{r})
$$

with $\omega_{\mathbf{q} \nu}$ the phonon frequency and $e_{\kappa \alpha, \nu}(\mathbf{q})$ the $\alpha$ th Cartesian component of the phonon eigenvector for atom $\kappa$ of mass $M_{\kappa}$ in the unit cell. $V_{\kappa \alpha, \mathbf{q}}(\mathbf{r})$ is the potential obtained from DFPT [16]. Following the approach adopted in the literature [6,11], $V_{\kappa \alpha, \mathbf{q}}$ is separated into short-range $(\mathrm{SR}, \mathcal{S})$ and $\mathrm{LR}(\mathcal{L})$ contributions: $V_{\kappa \alpha, \mathbf{q}}(\mathbf{r})=V_{\kappa \alpha, \mathbf{q}}^{\mathcal{S}}(\mathbf{r})+V_{\kappa \alpha, \mathbf{q}}^{\mathcal{L}}(\mathbf{r})$. The latter, $V^{\mathcal{L}}$, is supposed to include all the LR components so that $V^{\mathcal{S}}$ is smooth in $\mathbf{q}$ space and therefore tractable with Fourier interpolation. In the interpolation algorithm, $V^{\mathcal{L}}$ is first subtracted from the DFPT potentials, then the Fourier interpolation is performed on the SR part only. $V^{\mathcal{L}}$ evaluated at the arbitrary $\mathbf{q}$ point is finally added back [17]. In polar materials, the leading contribution to the LR part stems from the diverging Fröhlich-like potential [6,11]:
$V_{\kappa \alpha, \mathbf{q}}^{\mathcal{L}(F)}(\mathbf{r})=\frac{4 \pi}{\Omega} \sum_{\mathbf{G} \neq-\mathbf{q}} \frac{i\left(q_{\beta}+G_{\beta}\right) Z_{\kappa \alpha, \beta}^{*} e^{i\left(q_{\eta}+G_{\eta}\right)\left(r_{\eta}-\tau_{\kappa \eta}\right)}}{\left(q_{\delta}+G_{\delta}\right) \epsilon_{\delta \delta^{\prime}}^{\infty}\left(q_{\delta^{\prime}}+G_{\delta^{\prime}}\right)}$

with $\Omega$ the unit cell volume, $\mathbf{G}$ the reciprocal lattice vectors, $\mathbf{Z}_{\kappa}^{*}$ the Born effective charge tensor, $\boldsymbol{\epsilon}^{\infty}$ the high-frequency dielectric tensor, and $\boldsymbol{\tau}_{\kappa}$ the position of the $\kappa$ th atom in the unit cell. The summation over repeated indices $\left(\beta, \eta, \delta\right.$, and $\left.\delta^{\prime}\right)$ is implied in Eq. (2) and in the following, unless the sum is explicitly written.

Most investigations so far have focused on the treatment of Eq. (2). However, as discussed by Vogl [12] and derived in a DFPT context in our accompanying paper [17], a careful analysis of the asymptotic behavior of the scattering potential in the long-wavelength limit reveals the presence of additional LR terms besides Eq. (2). To the contrary of the $1 / q$ nature of Eq. (2), these additional terms are finite for $\mathbf{q} \rightarrow \mathbf{0}$ but their nonanalytic behavior (angular discontinuities) yields LR scattering potentials and associated $e$-ph matrix elements even when the dipole interaction given by Eq. (2) is properly accounted for. Both dipole and quadrupole terms can be included in the LR potential using the generalized expression [17]:

$$
V_{\kappa \alpha, \mathbf{q}}^{\mathcal{L}}(\mathbf{r})=\frac{4 \pi}{\Omega} \sum_{\mathbf{G} \neq-\mathbf{q}} \frac{i\left(q_{\beta}+G_{\beta}\right) Z_{\kappa \alpha, \beta}^{*}+\left(q_{\beta}+G_{\beta}\right)\left(q_{\gamma}+G_{\gamma}\right)\left(Z_{\kappa \alpha, \beta}^{*} v^{\left.\mathrm{HXC}, \mathcal{E}_{\gamma}(\mathbf{r})+\frac{1}{2} Q_{\kappa \alpha}^{\beta \gamma}\right)}\right.}{\left(q_{\delta}+G_{\delta}\right) \epsilon_{\delta \delta^{\prime}}^{\infty}\left(q_{\delta^{\prime}}+G_{\delta^{\prime}}\right)} e^{i\left(q_{\eta}+G_{\eta}\right)\left(r_{\eta}-\tau_{\kappa \eta}\right)},
$$

where $\mathbf{Q}_{\kappa \alpha}$ is the dynamical quadrupole tensor and $v^{\mathrm{HXC}, \mathcal{E}}$ is the change of the Hartree and exchange-correlation potential with respect to the electric field $\mathcal{E}$ in Cartesian coordinates. In Eq. (3), the term related to $\mathbf{Q}_{\kappa \alpha}$ is nonzero even in nonpolar semiconductors while the contribution associated with $\mathcal{E}$ is present only in systems with nonzero Born effective charges. A quadrupole can indeed appear even in nonpolar situations: when mirror and/or inversion symmetries are broken at a given atomic site, the charge induced by atomic motion can acquire an inversion-even and hence quadrupolar contribution. This quadrupolar effect is even present in elemental crystals with vanishing dipoles. This effect is illustrated in Fig. 1(a) reporting the variation of the charge density induced by a single atomic displacement in a 250-atoms supercell of Si. Such dynamical quadrupoles should not be confused with the static quadrupolar moment that characterizes certain molecules, e.g., $\mathrm{CO}_{2}$ [18]. Regardless of its static or dynamic nature, a localized charge quadrupole results in a LR scattering potential that extends over many unit cells of the unperturbed crystal, see Fig. 1(b). In the region around the displaced atom, this term is relatively small compared to the SR part but it becomes the dominant contribution at large distances. Its anisotropic and macroscopic character in real space determines the $\mathbf{q} \rightarrow \mathbf{0}$ behavior of the $e$-ph matrix elements in nonpolar materials, and constitutes an important contribution in polar materials. By contrast, the $v^{\mathrm{HXC}, \mathcal{E}}$ contribution is a secondary, indirect effect of the Fröhlich interaction: a local-field potential modification due to the charge density change induced by the Fröhlich dipolar electric field. Thus, it vanishes when $\mathbf{Z}_{\kappa}^{*}$ vanishes. (a)

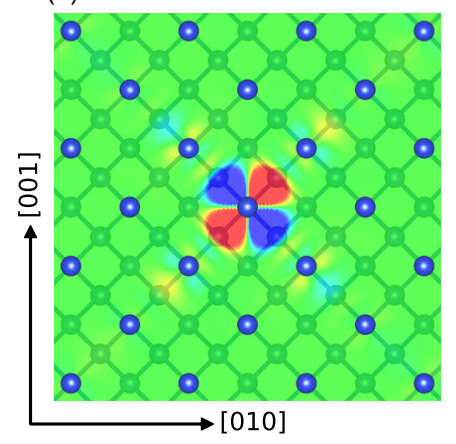

(b)

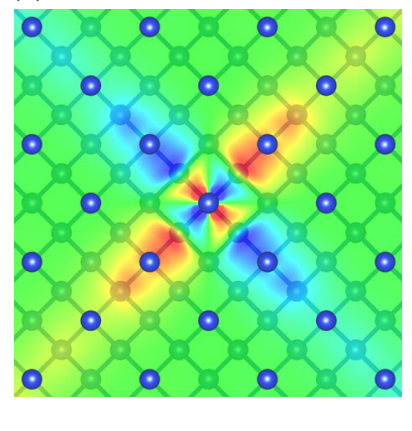

FIG. 1. (a) Electronic charge density variation induced by a single atomic displacement in $\mathrm{Si}$, along the [100] direction of the conventional cell (perpendicular to the represented plane). Negative (positive) variations are shown in red (blue), while green regions correspond to absolute values smaller than $10 \%$ of the in-plane maximum. (b) Scattering potential induced by the atomic displacement with the same color map as in (a). SR dipolelike contributions are absent in this plane [19]. 
There is no a priori rigorous justification for ignoring LR anisotropic contributions to the $e$-ph scattering generated by the quadrupolar terms. This is especially critical because $e$-ph properties are rather sensitive to the behavior of the potential in the macroscopic regime. We first gauge the importance of these effects on the $e$-ph scattering potentials. The effects on electron mobilities are discussed afterwards. Our study is based on a new $e$-ph implementation in ABINIT detailed in our accompanying paper [17]. The numerical values of $\mathbf{Q}_{\kappa \alpha}$ are computed using the recent implementation of Royo et al. [13], now integrated with the $e$-ph part of ABINIT [17,23-25]. Following previous works $[26,27]$, we treat the electron wave functions exactly and employ a Fourier-transform-based scheme for the scattering potentials [26]. This avoids a transformation to localized orbitals, and has the important advantage of being systematic and automatic.

In Figs. 2(a) and 2(b) we plot the unit-cell averaged lattice-periodic part of the scattering potential,

$$
\bar{V}_{\kappa \alpha, \mathbf{q}}=\frac{1}{\Omega} \int_{\Omega} d \mathbf{r} V_{\kappa \alpha, \mathbf{q}}(\mathbf{r}) e^{-i \mathbf{q} \cdot \mathbf{r}},
$$

for selected atomic perturbations in $\mathrm{Si}$ and $\mathrm{GaAs}$, along a high-symmetry q path. The exact DFPT results (blue lines) are compared with those obtained with the models with (green) or without (red) quadrupole corrections in Eq. (3) [19]. In Si, the Born effective charges are zero and the imaginary part of the potential does not diverge for $\mathbf{q} \rightarrow \mathbf{0}$ [see dashed lines in Fig. 2(a)]. In GaAs, the Fröhlich-like model in Eq. (2) correctly describes the divergence of the imaginary part of the potential close to $\Gamma$ [see red dashed line in Fig. 2(b)]. In both materials, however, the real part of the potential (solid lines) presents discontinuities for $\mathbf{q} \rightarrow \mathbf{0}$. Note that the Fröhlich term alone completely misses this nonanalytic behavior. On the contrary, if the quadrupolar contributions are included through Eq. (3), the LR model reproduces these discontinuities as shown by the solid green lines in Figs. 2(a) and 2(b). Figure 2(c) shows the real part of the average of the scattering potential in $\mathrm{Si}$ interpolated from a $9 \times 9 \times 9 \mathbf{q}$-point grid onto the same $\mathbf{q}$ path as in Fig. 2(a). If the LR quadrupolar terms are not removed from the input DFPT potentials, Fourier aliasing introduces unphysical sharp oscillations for small $\mathbf{q}$ (see red line, FI). The correct behavior is obtained only when quadrupolar contributions are properly treated (see green line, $\mathrm{FI}+Q$ ). In the latter case, small oscillations between the $a b$ initio $\mathbf{q}$ points are still visible when a $9 \times 9 \times 9 \mathbf{q}$ mesh is used. However, convergence studies [19] reveal that these wiggles have a limited effect on the final electron mobility (less than $0.5 \%$ difference between the mobility obtained for a $9 \times 9 \times 9$ and an $18 \times 18 \times 18$ ab initio $\mathbf{q}$ mesh) and they can be converged away.

It is worth stressing that these considerations hold for any approach employing Fourier-based interpolations.
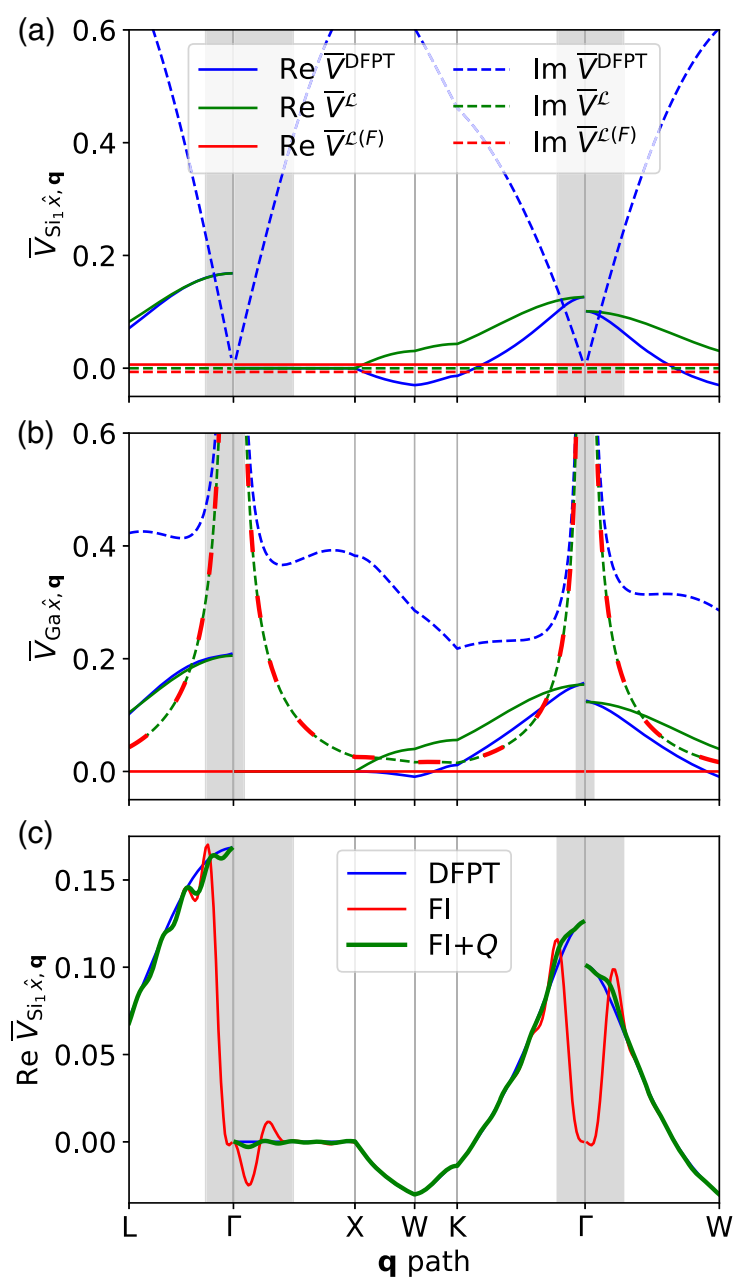

FIG. 2. Comparison of the unit-cell averaged exact DFPT potentials ( $\bar{V}^{\text {DFPT }}$, blue lines) with the models of Eqs. (2) $\left(\bar{V}^{\mathcal{L}(F)}\right.$, red lines) and (3) $\left(\bar{V}^{\mathcal{L}}\right.$, green lines) in (a) Si and (b) GaAs. We consider the first reduced component $\hat{x}$ of the DFPT potentials for (a) the $\mathrm{Si}$ and (b) the $\mathrm{Ga}$ atoms located at $(0,0,0)$. In (a), the red lines have been slightly shifted upwards and downwards for readability. (c) Fourier interpolation of the potentials shown in (a) with $(\mathrm{FI}+Q)$ or without $(\mathrm{FI})$ the treatment of the quadrupolar interaction, as explained in the text. The potentials are given in hartree. The path has been sampled with $278 \mathbf{q}$ points. The gray areas around $\Gamma$ represent the regions relevant for intravalley scattering, as dictated by Eq. (5).

The discontinuity of the matrix elements at $\Gamma$ and the discrepancy between the interpolant and the exact DFPT results in the region around $\Gamma$ have already been noticed for $\mathrm{Si}$ and diamond [8]. The resulting error was considered harmless under the assumption that it is always possible to improve the accuracy of the Fourier interpolation by densifying the initial $a b$ initio q mesh [8]. Unfortunately, this assumption does not hold in the presence of such LR behavior: using a denser DFPT mesh localizes the sharp oscillations of the potential in a slightly smaller region around $\mathbf{q}=\Gamma$ [see red curve in Fig. 2(c)], but their amplitude is proportional to the discontinuity [19]. In the 
Supplemental Material [19], we show that treating the quadrupole interaction is also important when using a Wannier-based interpolation scheme. This has also been recently confirmed by Jhalani et al. [28].

At this point, we can quantify the error introduced by these spurious oscillations in the final physical results, and the importance of the quadrupolar interactions. Because of intravalley transitions, the region around $\Gamma$ (where the LR interactions dominate) is usually one of the most important for the description of $e$-ph scattering processes $[17,19]$. In Fig. 2, the region around $\Gamma$ relevant for intravalley scattering is represented by gray areas. An accurate description in this region is crucial for reliable calculations of the phononinduced electron linewidths,

$$
\begin{aligned}
\tau_{n \mathbf{k}}^{-1}= & 2 \pi \sum_{m, \nu} \int_{\mathrm{BZ}} \frac{d \mathbf{q}}{\Omega_{\mathrm{BZ}}}\left|g_{m n \nu}(\mathbf{k}, \mathbf{q})\right|^{2} \\
& \times\left[\left(n_{\mathbf{q} \nu}+f_{m \mathbf{k}+\mathbf{q}}\right) \delta\left(\varepsilon_{n \mathbf{k}}-\varepsilon_{m \mathbf{k}+\mathbf{q}}+\omega_{\mathbf{q} \nu}\right)\right. \\
& \left.+\left(n_{\mathbf{q} \nu}+1-f_{m \mathbf{k}+\mathbf{q}}\right) \delta\left(\varepsilon_{n \mathbf{k}}-\varepsilon_{m \mathbf{k}+\mathbf{q}}-\omega_{\mathbf{q} \nu}\right)\right],
\end{aligned}
$$

with $\Omega_{\mathrm{BZ}}$ the BZ volume, $n_{\mathbf{q} \nu}$ and $f_{m \mathbf{k}+\mathbf{q}}$ the Bose-Einstein and Fermi-Dirac occupation functions, and $\varepsilon_{n \mathbf{k}}$ the energy of the electronic state $n \mathbf{k}$. These linewidths are needed to compute (phonon-limited) carrier mobilities within the selfenergy relaxation time approximation $[1,17,29,30]$ :

$$
\mu_{e, \alpha \beta}=\left.\frac{-1}{\Omega n_{e}} \sum_{n} \int \frac{d \mathbf{k}}{\Omega_{\mathrm{BZ}}} \mathrm{v}_{n \mathbf{k}, \alpha} \mathrm{v}_{n \mathbf{k}, \beta} \tau_{n \mathbf{k}} \frac{\partial f}{\partial \varepsilon}\right|_{\varepsilon_{n \mathbf{k}}},
$$

where $n_{e}$ is the electron concentration and $\mathrm{v}_{n \mathbf{k}, \alpha}$ is the $\alpha$ th component of the velocity operator [17]. Splitting the sum over $\nu$ in Eq. (5), one can obtain the partial mobility limited by a single phonon mode. Figure 3 reports the error made

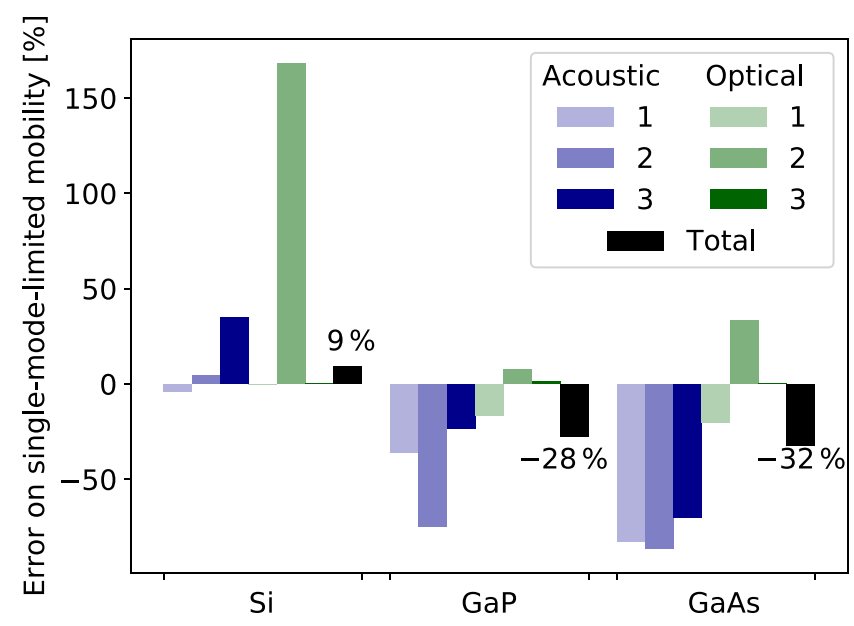

FIG. 3. Error on the single-phonon-mode limited mobility when the quadrupole interaction is not correctly treated in $\mathrm{Si}$, $\mathrm{GaP}$, and $\mathrm{GaAs}$, for acoustic (blue) and optical (green) modes. The error on the total mobility is given in black. on these single-phonon-mode limited mobilities in $\mathrm{Si}, \mathrm{GaP}$, and $\mathrm{GaAs}$ if quadrupole interactions are not properly treated when interpolating the potentials on the fine $\mathbf{q}$ mesh necessary for convergence [19]. The initial DFPT $\mathbf{q}$ meshes are $9 \times 9 \times 9$ for $\mathrm{Si}$ and $\mathrm{GaP}$, and $6 \times 6 \times 6$ for GaAs. We note that these errors are not significantly reduced by densifying the initial mesh if quadrupole interactions are not treated [19]. In Si, the quadrupole interaction mainly affects one of the optical modes. The effect on acoustic modes is much smaller, as expected from the quadrupole acoustic sum rule for nonpolar semiconductors and already stressed by Vogl [12]. In contrast, in $\mathrm{GaP}$ and particularly in GaAs, the acoustic modes are the most affected. This is also expected since these modes are dominated by the piezoelectric coupling related to acoustic modes [12]. When considering the scattering by all phonon modes, the error on the total mobility is around $10 \%$ in $\mathrm{Si}$ and goes up to $32 \%$ in GaAs. The larger error in GaAs is linked to its smaller effective mass and the presence of a single valley at $\Gamma$ hence most of the scattering for electrons close to the band edge is intravalley with small q, see Fig. 2. This shows the importance of the quadrupole interaction in understanding the physics of all classes of $e$-ph scattering. These errors on mobilities can be even more significant than many-body effects (within the transport formalism used in this work). Indeed, according to Poncé et al. GW corrections of the KS band energies of $\mathrm{Si}$ increase the electron mobility by $5 \%$ [30]. Electron-phonon calculations are usually performed starting from coarse $\mathbf{q}$ meshes in order to limit both the number of DFPT computations and the cost of the interpolation itself, which quickly increases with the number of $\mathbf{q}$ points in the initial ab initio mesh. It is thus not surprising that this behavior has been largely overlooked so far.

We can also gauge the relative importance of the dynamical quadrupole and the electric-field terms in Eq. (3). In nonpolar systems, $\mathbf{Q}_{\kappa \alpha}$ is the only additional quadrupolar term in the LR scattering potential. In the polar systems investigated so far, we observe that $\mathbf{Q}_{\kappa \alpha}$ gives a larger contribution to Eq. (3) than the $\mathcal{E}$ term [19]. For instance, ignoring the $\mathcal{E}$ term changes the electron mobility in $\mathrm{GaAs}(\mathrm{GaP})$ by $0.1 \%(0.01 \%)$. In $\mathrm{GaAs}$ and $\mathrm{GaP}$, one could speculate that the $\mathcal{E}$ term is negligible for acoustic modes, where the Fröhlich contribution exactly vanishes. Additionally, $v^{\mathrm{HXC}, \mathcal{E}}(\mathbf{r})$ oscillates on the scale of the interatomic distances, and couples more weakly to the electronic orbitals than a macroscopic potential shift. In the ionic limit, for example, one can expect $v^{\mathrm{HXC}, \mathcal{E}}(\mathbf{r})$ to be antisymmetric around each atomic site, with a vanishing diagonal matrix element for the corresponding symmetric electronic orbitals. Generalizations of the predominance of $\mathbf{Q}_{\kappa \alpha}$ to other systems should be done with care. For example, in many crystals without free Wyckoff parameters (e.g., rocksalt or cubic perovskite) the quadrupole tensor vanishes identically; thus, in such materials the $\mathcal{E}$ term 
remains the only source of nonanalytic behavior in the firstorder potential besides the Fröhlich divergence and could lead to interesting physics. Note that this term is absent in the contemporaneous analysis by Jhalani et al. [28].

We also note that $\mathbf{Q}_{\kappa \alpha}$ introduces additional dipolequadrupole and quadrupole-quadrupole terms at the dynamical matrix level, which have to be considered for accurate phonon spectra, especially for small $\mathbf{q}$ where artificial vibrational instabilities may appear [14,31]. These higher-order terms have also been included in our calculations. In $\mathrm{Si}, \mathrm{GaAs}$, and $\mathrm{GaP}$, they only change the mobility by $\sim 1 \%$, which is smaller than the effect of the quadrupole potential, but still larger than the effect of the electric-field induced local potential. However, we cannot exclude that these contributions might be larger for piezoelectric materials, in which spurious instabilities may appear in the phonon dispersion [31]. More details about the effects associated with the electric-field induced local potential, to the dipole-quadrupole and the quadrupole-quadrupole interactions in the dynamical matrix are discussed in the Supplemental Material [19].

In conclusion, we have included the treatment of quadrupolar fields, beyond the Fröhlich interaction, in the firstprinciples electron-phonon vertex for semiconductors. By their LR nature, a proper treatment of the quadrupolar fields is necessary for the accurate description of $e$-ph quantities such as the scattering potential and carrier mobilities. Without treating quadrupolar interactions, large errors from $10 \%$ to $30 \%$ can be obtained in evaluating the mobilities of polar and nonpolar semiconductors such as $\mathrm{Si}, \mathrm{GaP}$, or GaAs. Taking into account very recent results on $\mathrm{GaN}$ and $\mathrm{PbTiO}_{3}$ [28], we expect these additional contributions to be relevant in a wide variety of materials. For instance, the quadrupolar response is always present in piezoelectric materials [13], where the piezoelectric constants are uniquely defined by the dipole and quadrupole moments [15]. These LR physical effects lead to important corrections which can be even more significant than quasiparticle corrections. Since LR macroscopic interactions play a key role in semiconductor physics, we believe that the quest for accurate $a b$ initio descriptions of phonon-limited carrier mobilities and other $e$-ph related properties should start from a proper treatment of these physical phenomena.

G. B., G.-M. R., and G.H. acknowledge financial support from F.R.S.-FNRS. H. P. C. M. acknowledges financial support from F.R.S.-FNRS through the PDR Grants HTBaSE (T.1071.15). M. G., M. J. V., and X. G. acknowledge financial support from F.R.S.-FNRS through the PDR Grants AIXPHO (T.0238.13) and ALPS (T.0103.19). M. J. V. thanks FNRS and ULiege for a sabbatical grant in ICN2. M. S. and M. R. acknowledge the support of Ministerio de Economia, Industria y Competitividad (MINECO-Spain) through Grants No. MAT2016-77100C2-2-P and No. SEV-2015-0496; of Generalitat de Catalunya (Grant No. 2017 SGR1506); and of the
European Research Council (ERC) under the European Union's Horizon 2020 research and innovation program (Grant Agreement No. 724529). The present research benefited from computational resources made available on the Tier-1 supercomputer of the Fédération WallonieBruxelles, infrastructure funded by the Walloon Region under Grant Agreement No. 1117545.

Note added-Recently, we became aware of a related work by another group that reaches similar conclusions about the importance of the dynamical quadrupole term to obtain an accurate physical description of $e$-ph interactions [28].

*Corresponding author. geoffroy.hautier@uclouvain.be

[1] F. Giustino, Rev. Mod. Phys. 89, 015003 (2017).

[2] W. Kohn and L. Sham, Phys. Rev. 140, A1133 (1965).

[3] X. Gonze and C. Lee, Phys. Rev. B 55, 10355 (1997).

[4] S. Baroni, S. de Gironcoli, A. Dal Corso, and P. Giannozzi, Rev. Mod. Phys. 73, 515 (2001).

[5] F. Giustino, M. L. Cohen, and S. G. Louie, Phys. Rev. B 76, 165108 (2007)

[6] J. Sjakste, N. Vast, M. Calandra, and F. Mauri, Phys. Rev. B 92, 054307 (2015).

[7] S. Poncé, E. R. Margine, C. Verdi, and F. Giustino, Comput. Phys. Commun. 209, 116 (2016).

[8] L. A. Agapito and M. Bernardi, Phys. Rev. B 97, 235146 (2018).

[9] M. Born and K. Huang, Dynamical Theory of Crystal Lattices (Oxford University Press, Oxford, 1954).

[10] H. Fröhlich, Adv. Phys. 3, 325 (1954).

[11] C. Verdi and F. Giustino, Phys. Rev. Lett. 115, 176401 (2015).

[12] P. Vogl, Phys. Rev. B 13, 694 (1976).

[13] M. Royo and M. Stengel, Phys. Rev. X 9, 021050 (2019).

[14] M. Stengel, Phys. Rev. B 88, 174106 (2013).

[15] R. M. Martin, Phys. Rev. B 5, 1607 (1972).

[16] More specifically, $\quad V_{\kappa \alpha, \mathbf{q}}(\mathbf{r}) \equiv e^{i \mathbf{q} \cdot \mathbf{r}} \partial_{\kappa \alpha, \mathbf{q}} v^{\mathrm{KS}}(\mathbf{r})$, with $\partial_{\kappa \alpha, \mathbf{q}} v^{\mathrm{KS}}(\mathbf{r})$ the lattice-periodic part computed with DFPT [17].

[17] G. Brunin, H. P. C. Miranda, M. Giantomassi, M. Royo, M. Stengel, M. J. Verstraete, X. Gonze, G.-M. Rignanese, and G. Hautier, companion paper, Phys. Rev. B 102094308 (2020).

[18] C. Graham, J. Pierrus, and R. Raab, Mol. Phys. 67, 939 (1989).

[19] See Supplemental Material at http://link.aps.org/supplemental/ 10.1103/PhysRevLett.125.136601 for more information about computational details and results, which includes Refs. [20-22].

[20] M. J. Van Setten, M. Giantomassi, E. Bousquet, M. J. Verstraete, D. R. Hamann, X. Gonze, and G.-M. Rignanese, Comput. Phys. Commun. 226, 39 (2018).

[21] D. R. Hamann, Phys. Rev. B 88, 085117 (2013).

[22] N. Troullier and J. L. Martins, Phys. Rev. B 43, 1993 (1991); J. P. Perdew and Y. Wang, Phys. Rev. B 45, 13244 (1992); D. M. Ceperley and B. J. Alder, Phys. Rev. Lett. 45, 566 (1980). 
[23] X. Gonze, F. Jollet, F. A. Araujo, D. Adams, B. Amadon, T. Applencourt, C. Audouze, J.-M. Beuken, J. Bieder, A. Bokhanchuk et al., Comput. Phys. Commun. 205, 106 (2016).

[24] X. Gonze, B. Amadon, G. Antonius, F. Arnardi, L. Baguet, J.-M. Beuken, J. Bieder, F. Bottin, J. Bouchet, E. Bousquet et al., Comput. Phys. Commun. 248, 107042 (2020).

[25] A. H. Romero, D. C. Allan, B. Amadon, G. Antonius, T. Applencourt, L. Baguet, J. Bieder, F. Bottin, J. Bouchet, E. Bousquet et al., J. Chem. Phys. 152, 124102 (2020).

[26] A. Eiguren and C. Ambrosch-Draxl, Phys. Rev. B 78, 045124 (2008).
[27] L. Chaput, A. Togo, and I. Tanaka, Phys. Rev. B 100, 174304 (2019).

[28] V. A. Jhalani, J.-J. Zhou, J. Park, C. E. Dreyer, and M. Bernardi, following Letter, Phys. Rev. Lett. 125, 136602 (2020); J. Park, J.-J. Zhou, V. A. Jhalani, C. E. Dreyer, and M. Bernardi, companion paper, Phys. Rev. B 102, 125203 (2020).

[29] G. K. H. Madsen, J. Carrete, and M. J. Verstraete, Comput. Phys. Commun. 231, 140 (2018).

[30] S. Poncé, E. R. Margine, and F. Giustino, Phys. Rev. B 97, 121201(R) (2018).

[31] M. Royo, K. R. Hahn, and M. Stengel, https://arxiv.org/abs/ 2004.08875 